\title{
Relationship between malnutrition-inflammation complex syndrome and fluid balance in maintenance hemodialysis patients
}

\begin{abstract}
The aim of this study was to assess the prevalence of malnutrition-inflammation complex syndrome (MICS) and determine sensitivity and specificity of MICS markers in hemodialysis (HD) patients depending on volume status. The study included 124 patients undergoing adequate HD. The patients were distributed based on IDWG (interdialytic weight gain)/ DBW (dry body weight) and IDWG/TBW (total body water) ratios in 4 groups: 1 - IDWG/ DBW $\leq 5 \%$ and IDWG/TBW $\leq 10 \% ; 2-$ IDWG/DBW $\leq 5 \%$ and IDWG/TBW $>10 \% ; 3$ - IDWG/DBW $>5 \%$ and IDWG/TBW $\leq 10 \%$, and 4 - IDWG/DBW $>5 \%$ and IDWG/ TBW $>10 \%$. MICS was evaluated based on malnutrition and inflammation markers. Data were analyzed with ANOVA, chi-square test, R.O.C. curve and Odds ratio, using SPSS software; $p<0.05$ was considered statistically significant. A significant difference was found in patients' distribution pattern according to IDWG/DBW and IDWG/TBW ratios ( $p<0.0001$ ). The rate of volume overload, represented with IDWG/TBW $>10 \%$, was significantly higher in patients in groups 3 and 4. Groups also differed in age ( $F=3.3, p$ $<0.02)$ and residual diuresis $(\mathrm{F}=2.3, \mathrm{p}<0.05)$. In spite of different volume status, systolic and diastolic blood pressure did not differ significantly between groups. Serum sodium ( $\mathrm{p}$ $<0.043)$ and chloride $(p<0.035)$ differed significantly between groups - patients in group 1 had highest, while patients in group 4 had lowest sodium and chloride levels. CRP levels did not differ significantly between groups. The highest prevalence of MICS was found in group 4 (up to $30 \%)$. BMI had strongest predictive value for MICS in groups 1 and $4(\mathrm{p}=$ 0.000 and $\mathrm{p}=0.002$ respectively), followed by malnutrition-inflammation score, MIS ( $\mathrm{p}=$ 0.053 and $p=0.057$ respectively). Ferritin and BMI were the strongest predictors of MICS in group $3(p=0.023$ and $p=0.051$ respectively). Patients in group 4 had three times higher chances to have MICS then those in group $3(\mathrm{OR}=3$, CI: $1.7645<$ O.R. $<5.1007)$. In this study, the excessive volume overload implied increased risk and high prevalence of MICS. Low BMI and MIS appeared to be the most sensitive predictors of MICS, while low BMI also represented strong risk factor for disturbed fluid balance.
\end{abstract}

Keywords: hemodialysis, fluid balance, hypervolemia, inflammation, malnutrition
Volume 4 Issue 5 - 2017

\author{
Vlastimir Vlatković,' Jasna Trbojević- \\ Stanković, ${ }^{2}$ Biljana Stojimirović ${ }^{3}$ \\ 'University Clinical Center of the RepublikaSrpska, Bosnia \& \\ Herzegovina \\ ${ }^{2}$ Dialysis Department, University of Belgrade and Clinic of \\ Urology, Serbia \\ ${ }^{3}$ University of Belgrade and Institute for Urology and \\ Nephrology, Serbia
}

Correspondence: VlastimirVlatković, University clinical center of the RepublikaSrpska, 78000 Banja Luka, Republic of Srpska, Bosnia \& Herzegovina, Tel +387 66707089 ,

Email wlastimir@hotmail.com

Received: November 18,2016 | Published: May 09, 2017

\begin{abstract}
Abbreviations:ALP,alkaline mass index:BP,blood preasure; PCI, phatse;BMI, body index;CRP,standard C-Reactive protein; DBW,dry body weight; ESRD, end-stage renal disease, $\mathrm{Fe}^{2+}$, serum iron; HCT,hematocrit;HD, hemodialysis;HDL,high density lipoproteins; HGB, hemoglobin;IDWG, interdialyticweight gain;iPTH,intact parathyroid hormone;LDL,low density lipoproteins;LDL/ HDL,atherogenicity index; MIA, malnutrition-inflammation and atherosclerosis syndrome; MICS, malnutrition-inflammationcomplex syndrome; MIS,malnutrition-inflammation score;MIS,malnutritioninflammation score; OR,odds ratio; SGOT,glutamic oxaloacetic transaminase;SGPT,glutamic pyruvic transaminase; $\mathrm{spK} / \mathrm{V}$, single pool $\mathrm{Kt} / \mathrm{V}$;TBW,total body water;TIBC,total iron binding capacity;TSAT,transferrin saturation index;URR,urea reduction ratio
\end{abstract}

\section{Introduction}

End-stage renal disease (ESRD) patients undergoing maintenance hemodialysis (HD) have a high prevalence of protein-energy malnutritionandinflammation.' Since these conditions tend to occur concomitantly and coexist in individuals with ESRD, the terms malnutrition-inflammation complex syndrome (MICS) or malnutrition-inflammation, and atherosclerosis (MIA) syndrome have been proposed to indicate the combination of these two conditions in HD patients. ${ }^{1,2}$ Possible causes of MICS include: anorexia and low energy intake, oxidative and carbonyl stress, protein and calorie loss during $\mathrm{HD}$, uremic toxins, impaired pro-inflammatory cytokines clearance, comorbidities, volume overload and dialysis-related factors. ${ }^{2,3}$ MICS prevalence of 30-60\% was reported in Northern American and European HD patients. Dialysis patients in Asian countries may have lower prevalence of inflammation, possibly caused by genetic factors or environmental entities, including diet. ${ }^{2-4}$

There is increasing evidence that volume overload may be a stimulus for systemic inflammation. Patients with fluid overload, such as those with heart failure, present higher endotoxin levels than the normovolemic ones. ${ }^{5,6}$ After the treatment with diuretics, a significant reduction in endotoxin levels was observed, indicating that hypervolemia and/or heart failure are associated with immune activation. Furthermore, a negative correlationreported between volume overload and serum albumin (negative acute-phase protein and nutritional marker) in HD patients is not only a result of dilution, but also of increased serum endotoxin and cytokine levels and decreased albumin synthesis due to hyperhydration. ${ }^{7,8}$ Nearly $90 \%$ of ESRD 
patients starting HD are hypertensive, ${ }^{9}$ mainly due to high plasma sodium level and extracellular volume expansion. ${ }^{6}$ Owing to the high hydraulic permeability of the cell membrane, water moves freely between intracellular and extracellular compartment. This maintains an equal osmolality in all fluid compartments of the body. ${ }^{9}$ Due to the intermittent nature of $\mathrm{HD}$, the patient oscillates between hypervolemic state, just before the session, and normovolemic, "dry", state at the end of the session. ${ }^{9}$ This has led to the concept of dry body weight (DBW), a crucial component of dialysis adequacy. DBW reflects the lowest post-dialysis weight at which the patient is and remains normotensive until the next dialysis in spite of the interdialytic fluid retention and without anti-hypertensive medications. ${ }^{9}$

Volume overload mostly depends on interdialytic weight gain (IDWG) and sodium consumption. ${ }^{10}$ It causes tissue hyperhydration, disturbed electrolyte balance and hypertension. ${ }^{11}$ High IDWG is strongly associated with increase of blood pressure (BP), leading to left ventricular hyperthrophy. ${ }^{10,11}$ Identical IDWG results in different volume overload in individual HD patients, since total body water (TBW) and volume distribution depend on patient's body mass, age and sex. ${ }^{12}$ The aims of this study were to assess the prevalence of MICS and determine sensitivity and specificity of MICS markers in maintenance HD patients with different volume status.

\section{Materials and methods}

\section{Patients}

A total of 124 Caucasian patients, 72 males and 52 females, mean age $54.8 \pm 13.1$ years, from the International Dialysis Center in Banja Luka, were enrolled in the research. The study was conducted in compliance with the Helsinki Declaration and the study protocol was approved by the Ethics Committee of the University School of Medicine in Belgrade. All patients gave their informed consent for participation in this research. Patients dialyzed less than three times per week and those with amputated extremities were not included. The patients were distributed in 4 groups according to IDWG/DBW and IDWG/TBW ratios: group $1-$ IDWG/DBW $\leq 5 \%$ and IDWG/TBW $\leq 10 \%$; group $2-\mathrm{IDWG} / \mathrm{DBW} \leq 5 \%$ and $\mathrm{IDWG} / \mathrm{TBW}>10 \%$; group $3-$ IDWG/DBW $>5 \%$ and IDWG/TBW $\leq 10 \%$ and group $4-$ IDWG/ DBW $>5 \%$ and IDWG/TBW $>10 \%$; (Figure 1). Volume overload was defined as $>10 \%$ increase of TBW. Demographic and dialysis-related characteristics of the study population are presented in Table 1. Most patients $(97,65.3 \%)$ have been on dialysis for less than 5 years, but 7 have been on regular HD for over 15 years. All were on standard bicarbonate $\mathrm{HD}$, performed thrice weekly, using machinery with controlled ultra filtration andpolysulfone high and low flux dialysers. Nineteen patients, 8 women and 11 men $\left(\chi^{2}=0.005, p>0.05\right)$, had diabetes.

All patients received phosphate binders (calcium carbonate, calcium acetate and sevelamer hydrochloride), adequate substitutive therapy (recombined human erythropoietin, intravenous iron, vitamins $\mathrm{B}$ and $\mathrm{C}$ ) and vitamin $\mathrm{D}$ analogues if needed. Fifty-six patients (38.1\%) were on ACE inhibitors, 40 (27.2\%) were taking calciumchannel blockers and 10 patients $(6.8 \%)$ were treated with statins.

\section{Study protocol}

Basic epidemiological data were collected from anamnesis and medical records. DBW was determined by the clinical method, meaning undertaking systematic step-by-step lowering of post-HD weight until hypotension and/or cramps occurred. ${ }^{9}$ Ultra filtration was carefully controlled and planned for each session and BP continuously monitored. Patients were measured on the same, rigorously calibrated, scale, always under the same conditions (hours, clothing). TBW was calculated using the Watson formula,based on gender, age, height and body weight. ${ }^{12}$ Body Mass Index(BMI) was calculated according to the Quetelet equation as the ratio of weight $(\mathrm{kg})$ and squared size $\left(\mathrm{m}^{2}\right) \cdot{ }^{13}$ IDWG was defined as the difference between body weight before the first weekly HD session and DBW. It was assessed before the first weekly HD session, after the longest interdialytic period. At the same time, patients were thoroughly examined and no signs of pulmonary or peripheral edema were found. BP was measured with mercury column sphygmomanometer by a trained clinician, just before beginning of the first weekly HD session.

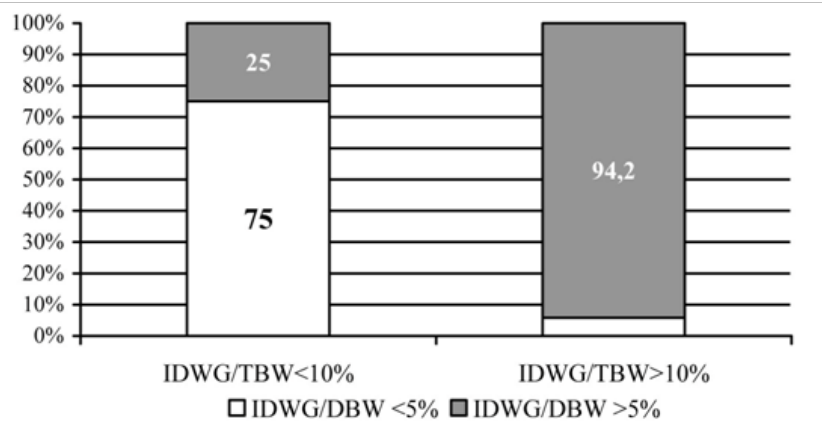

Figure I Distribution of patients according to interdialytic weight gain/total body water (IDWG/TBW) and interdialytic weight gain/dry body weight (IDWG/DBW) ratios.

Venous blood samples were drawn immediately before and immediately after the first dialysis session of the week. Leukocyte and platelet count were determined by impedance method, hemoglobin (HGB) with cyanmethemoglobin method on Cell Dyn 1700 counter. Hematocrit (HCT) and transferrin saturation index (TSAT) were calculated from the standard equations. ${ }^{14}$

Serum iron $\left(\mathrm{Fe}^{2+}\right)$ and total iron binding capacity (TIBC) were determined with Opton spectrophotometer. Serum ferritin was determined by immunochemistry MEIA technique, on Imx, Abbot machine.${ }^{14}$ Alcyon (Abbot) machine was used to determine: serum urea by urease glutamate dehydrogenase method; serum creatinine by Jaffe's method; uric acid by uricase method; serum albumin by spectrophotometry staining with bromcresol green;blood glucose with hexokinase; total cholesterol with cholesterol esteraze; triglycerides with lipase; high density lipoproteins (HDL) by precipitation; alkaline phosphatase (ALP), glutamic oxaloacetic transaminase (SGOT) and glutamic pyruvic transaminase (SGPT) by kinetic method; serum calcium by photometry and serum phosphates by photometric UV method. ${ }^{15}$ Low density lipoproteins (LDL) level was calculated from triglycerides, total cholesterol and HDL values. Intact parathyroid hormone (iPTH) was determined on autogenous counter, by immunoradiometric assayELSA-PTH, (CIS, France). ${ }^{16}$ Standard C-reactive protein (CRP) was measured by immunoturbidimetric method on Hitachi 912, Roche. Eventual laboratory errors were minimized by an internal control system. Dialysis dose was calculated based on mathematical models: urea reduction ratio (URR) and single pool Kt/V. URR was calculated from Lowrie's formula and Kt/V index from Daugirdas's formula. ${ }^{16}$ The severity of comorbidity was assessed by Charlson comorbidity index (CCI) without age component. The malnutrition - inflammation score (MIS) with 10 components was used as additional test for assessing MICS. ${ }^{17}$ The presence of MICS was defined with the following 5 variables: BMI $<20 \mathrm{~kg} / \mathrm{m}^{2}$, serum albumin $<40 \mathrm{~g} / \mathrm{L}$, ferritin $\geq 1000 \mathrm{umol} / \mathrm{L}, \mathrm{CRP}>8 \mathrm{ug} / \mathrm{dLandMIS} \geq 8$. 


\section{Statistical analysis}

The results are expressed as arithmetic mean \pm standard deviation. Patients' distribution according to IDWG/DBW and IDWG/TBW ratios was analyzed with $\chi^{2}$ goodness-of-fit test (Pearson product moment correlation coefficient) and test for homogenicity. ANOVA (F-test) was used to evaluate the differences between groups. Sensitivity and specificity of MICS parameters were determined with Receiver Operating Characteristics (ROC) analysis, while predictive strength of the chosen MICS parameters was assessed with Odds Ratio (OR). All data were analysed using Social Sciences (SPSS) software, Version 10.0 for Windows (SPSS, Chigaco, IL, USA). P value less then 0.05 was considered statistically significant.

\section{Results}

A statistically significant difference was found in patients' distribution pattern according to IDWG/DBW and IDWG/TBW

Table IDemographic and dialysis-related characteristics of study population ratios $\square \square^{2}=58.265, \mathrm{p}<0.0001$, (Figure 1). The rate of volume overload, as represented with IDWG/TBW $>10 \%$, was significantly higher in patients with IDWG/DBW $>5 \%$ than in those with IDWG/ DBW $\leq 5 \%$ ( $94.2 \%$ vs. $25 \%$; Figure 1$)$. All patients received adequate dialysis dose, according to URR and $\mathrm{Kt} / \mathrm{V}$ index (Table 1). Groups differed significantly in age $(\mathrm{p}<0.02$; Table 1$)$ and residual diuresis ( $\mathrm{p}<0.05$; Table 1). Patients in group 2 were oldest and had lowest residual diuresis. IDWG was lowest in group 1 and highest in group 4 ( $\mathrm{p}<0.0001$, Table 1$)$. No statistically significant difference was noted in systolic and diastolic BP between groups, in spite of different volume status (Table 1). All patients had HGB values within the recommended range for HD patients $(110-112 \mathrm{~g} / \mathrm{dL}) .{ }^{14}$ Patients in group 2 had highest HGB and HCT, but the difference was not statistically significant (Table 2). Transferrin saturation values were at lower threshold or insignificantly below recommended levels of 20$40 \%$ ((Table 2$).{ }^{14}$ Serum ferritin was within target range in all patients, with no significant differences between groups (Table 5).

\begin{tabular}{|c|c|c|c|c|c|c|c|c|}
\hline \multirow{2}{*}{ Item } & \multirow{2}{*}{ Group } & \multirow{2}{*}{$\mathbf{N}$} & \multirow{2}{*}{$\bar{X} \pm \mathbf{S D}$} & \multicolumn{2}{|c|}{$\mathbf{9 5} \%$ Interval } & \multirow{2}{*}{ Min } & \multirow{2}{*}{ Max } & \multirow{2}{*}{$\begin{array}{l}\text { F-test } \\
\text { P }\end{array}$} \\
\hline & & & & Upper & Lower & & & \\
\hline \multirow{5}{*}{ Age(years) } & 1 & 54 & $57.8 \pm 12$ & 54.4 & 61.1 & 23 & 82 & \multirow{5}{*}{$\begin{array}{l}F=3.3 \\
P<0.022\end{array}$} \\
\hline & 2 & 3 & $64 \pm 9.8$ & 39.5 & 88.5 & 56 & 75 & \\
\hline & 3 & 18 & $48.1 \pm 15.2$ & 40.5 & 55.6 & 22 & 72 & \\
\hline & 4 & 49 & $53.5 \pm 12.6$ & 49.9 & 57.1 & 30 & 78 & \\
\hline & Total & 124 & $54.8 \pm 13.1$ & 52.4 & 57.1 & 22 & 82 & \\
\hline \multirow{5}{*}{$\begin{array}{l}\text { Interdialytic weight } \\
\text { gain - IDWG (kg) }\end{array}$} & I & 54 & $2.8 \pm 0.8$ & 2.6 & 3 & 0.7 & 4.8 & \multirow{5}{*}{$\begin{array}{l}F=27.6 \\
P<0.000 I\end{array}$} \\
\hline & 2 & 3 & $3.5 \pm 0.4$ & 2.5 & 4.6 & 3.2 & 4 & \\
\hline & 3 & 18 & $3.6 \pm 0.5$ & 3.4 & 3.8 & 2.7 & 4.6 & \\
\hline & 4 & 49 & $4.1 \pm 0.7$ & 3.9 & 4.3 & 2.8 & 5.7 & \\
\hline & Total & 124 & $3.4 \pm 0.9$ & 3.3 & 3.6 & 0.7 & 5.7 & \\
\hline \multirow{5}{*}{$\begin{array}{l}\text { Dry body weight } \\
\text {-DBW (kg) }\end{array}$} & I & 54 & 70.3011 .99 & 67.03 & 73.58 & 43.50 & 104.50 & \multirow{5}{*}{$\begin{array}{l}F=6,679 \\
P<0,0001\end{array}$} \\
\hline & 2 & 3 & 76.3015 .06 & 38.88 & 113.72 & 65.00 & 93.40 & \\
\hline & 3 & 18 & 65.797 .96 & 61.84 & 69.75 & 53.50 & 81.00 & \\
\hline & 4 & 49 & 61.459 .95 & 58.59 & 64.31 & 41.50 & 91.00 & \\
\hline & Total & 124 & $66.30 \quad 11.49$ & 64.25 & 68.34 & 41.50 & 104.50 & \\
\hline \multirow{5}{*}{$\begin{array}{l}\text { Total body water - } \\
\text { TBW (L) }\end{array}$} & I & 54 & $36.69 \pm 6.24$ & 34.99 & 38.40 & 24.57 & 50.90 & \multirow{5}{*}{$\begin{array}{l}F=4,225 \\
P<0,007\end{array}$} \\
\hline & 2 & 3 & $34.54 \pm 4.10$ & 24.35 & 44.74 & 30.94 & 39.01 & \\
\hline & 3 & 18 & $38.35 \pm 4.58$ & 36.07 & 40.63 & 28.63 & 48.38 & \\
\hline & 4 & 49 & $33.55 \pm 5.46$ & 31.98 & 35.12 & 25.35 & 47.97 & \\
\hline & Total & 124 & $35.64 \pm 5.91$ & 34.59 & 36.69 & 24.57 & 50.90 & \\
\hline \multirow{5}{*}{$\begin{array}{l}\text { Duration of HD } \\
\text { session (Min) }\end{array}$} & I & 54 & $240.2 \pm 1.4$ & 239.8 & 240.6 & 240 & 250 & \multirow{5}{*}{ NS } \\
\hline & 2 & 3 & $240 \pm 0$ & 240 & 240 & 240 & 240 & \\
\hline & 3 & 18 & $240 \pm 0$ & 240 & 240 & 240 & 240 & \\
\hline & 4 & 49 & $240 \pm 0$ & 240 & 240 & 240 & 240 & \\
\hline & Total & 124 & $240.1 \pm 0.9$ & 239.9 & 240.2 & 240 & 250 & \\
\hline
\end{tabular}


Table Continues..

\begin{tabular}{|c|c|c|c|c|c|c|c|c|}
\hline \multirow{2}{*}{ Item } & \multirow{2}{*}{ Group } & \multirow{2}{*}{$\mathbf{N}$} & \multirow{2}{*}{$\bar{X} \pm \mathbf{S D}$} & \multicolumn{2}{|c|}{ 95\% Interval } & \multirow{2}{*}{ Min } & \multirow{2}{*}{$\operatorname{Max}$} & \multirow{2}{*}{$\begin{array}{l}\text { F-test } \\
\text { P }\end{array}$} \\
\hline & & & & Upper & Lower & & & \\
\hline \multirow{5}{*}{$\begin{array}{l}\text { Systolic blood } \\
\text { pressure before } \\
\mathrm{HD}(\mathrm{mmHg})\end{array}$} & I & 54 & $|30.4 \pm 2| .7$ & 124.4 & 136.3 & 80 & 180 & \multirow{5}{*}{ NS } \\
\hline & 2 & 3 & $106.7 \pm \mid 1.5$ & 78 & 135.4 & 100 & 120 & \\
\hline & 3 & 18 & $132.2 \pm 20.7$ & 121.9 & 142.5 & 80 & 160 & \\
\hline & 4 & 49 & $133.3 \pm 22$ & 126.9 & 139.6 & 100 & 190 & \\
\hline & Total & 124 & $|3| .2 \pm 2 \mid .7$ & 127.4 & I35.I & 80 & 190 & \\
\hline \multirow{5}{*}{$\begin{array}{l}\text { Diastolic blood } \\
\text { pressure before } \\
\mathrm{HD}(\mathrm{mmHg})\end{array}$} & I & 54 & $76.3 \pm 9.6$ & 73.7 & 78.9 & 60 & 90 & \multirow{5}{*}{ NS } \\
\hline & 2 & 3 & $66.7 \pm 11.5$ & 38 & 95.4 & 60 & 80 & \\
\hline & 3 & 18 & $77.2 \pm 10.2$ & 72.2 & 82.3 & 50 & 90 & \\
\hline & 4 & 49 & $77.1 \pm 10$ & 74.3 & 80 & 60 & 100 & \\
\hline & Total & 124 & $76.5 \pm 9.9$ & 74.8 & 78.3 & 50 & 100 & \\
\hline \multirow{5}{*}{$\begin{array}{l}\text { Residual } \\
\text { diuresis(ml/24 h) }\end{array}$} & I & 54 & $249.1 \pm 322.8$ & 161 & 337.2 & 0 & 1200 & \multirow{5}{*}{$\begin{array}{l}F=2.3 \\
P<0.05\end{array}$} \\
\hline & 2 & 3 & $66.7 \pm 57.7$ & 76.8 & 210.1 & 0 & 100 & \\
\hline & 3 & 18 & $305.6 \pm 469.6$ & 72 & 539.1 & 0 & 1500 & \\
\hline & 4 & 49 & $200 \pm 224.1$ & 135.6 & 264.4 & 0 & 1000 & \\
\hline & Total & 124 & $233.5 \pm 311.5$ & I78.I & 288.8 & 0 & 1500 & \\
\hline \multirow{5}{*}{ Single-pool Kt/V } & I & 54 & $1.4 \pm 0.2$ & 1.3 & 1.4 & 0.9 & 2 & \multirow{5}{*}{ NS } \\
\hline & 2 & 3 & $1.4 \pm 0.2$ & 0.7 & 2.2 & I.I & 1.6 & \\
\hline & 3 & 18 & $1.3 \pm 0.3$ & 1.2 & 1.5 & 0.9 & 2.2 & \\
\hline & 4 & 49 & $1.4 \pm 0.3$ & 1.3 & 1.5 & 0.8 & 2.1 & \\
\hline & Total & 124 & $1.4 \pm 0.3$ & 1.3 & 1.4 & 0.8 & 2.2 & \\
\hline \multirow{5}{*}{$\begin{array}{l}\text { Urea reduction ratio } \\
(\%)\end{array}$} & I & 54 & $67.1 \pm 6.9$ & 65.3 & 69 & 50 & 82 & \multirow{5}{*}{ NS } \\
\hline & 2 & 3 & $68 \pm 8.7$ & 46.3 & 89.7 & 58 & 74 & \\
\hline & 3 & 18 & $65.6 \pm 6.6$ & 62.3 & 68.8 & 53 & 83 & \\
\hline & 4 & 49 & $67.2 \pm 7.2$ & 65.1 & 69.2 & 46 & 82 & \\
\hline & Total & 124 & $66.9 \pm 6.9$ & 65.7 & 68.2 & 46 & 83 & \\
\hline
\end{tabular}

tnot significant

Table 2Basic laboratory tests I

\begin{tabular}{|c|c|c|c|c|c|c|c|c|}
\hline \multirow{2}{*}{ Item } & \multirow{2}{*}{ Group } & \multirow{2}{*}{$\mathbf{N}$} & \multirow{2}{*}{$\bar{X} \mathbf{\pm S D}$} & \multicolumn{2}{|c|}{$\mathbf{9 5 \%}$ Interval } & \multirow{2}{*}{ Min } & \multirow{2}{*}{ Max } & \multirow{2}{*}{$\begin{array}{l}\text { F-test } \\
\text { P }\end{array}$} \\
\hline & & & & Upper & Lower & & & \\
\hline \multirow{4}{*}{ Hematocrit (\%) } & I & 54 & $30.3 \pm 4.6$ & 29 & 31.6 & 12.7 & 40.9 & \multirow{4}{*}{$\mathrm{NS}^{\dagger}$} \\
\hline & 2 & 3 & $34.3 \pm 5.6$ & 20.3 & 48.3 & 30.6 & 40.8 & \\
\hline & 3 & 18 & $30.5 \pm 2.5$ & 29.2 & 31.7 & 25.9 & 35.7 & \\
\hline & Total & 124 & $30.4 \pm 4.4$ & 29.6 & 31.2 & 12.7 & 40.9 & \\
\hline \multirow{2}{*}{ Hemoglobin $(g / d l)$} & I & 54 & $111.6 \pm 16.2$ & 107.2 & 116 & 47 & 145 & \multirow{2}{*}{ NS } \\
\hline & 2 & 3 & $126.7 \pm 17.8$ & 82.5 & 170.8 & 114 & 147 & \\
\hline
\end{tabular}


Table Continues...

\begin{tabular}{|c|c|c|c|c|c|c|c|c|}
\hline \multirow{2}{*}{ Item } & \multirow{2}{*}{ Group } & \multirow{2}{*}{$\mathbf{N}$} & \multirow{2}{*}{$\bar{X} \pm \mathbf{S D}$} & \multicolumn{2}{|c|}{ 95\% Interval } & \multirow{2}{*}{ Min } & \multirow{2}{*}{ Max } & \multirow{2}{*}{$\begin{array}{l}\text { F-test } \\
\text { P }\end{array}$} \\
\hline & & & & Upper & Lower & & & \\
\hline \multirow{5}{*}{$\begin{array}{l}\text { Transferrin } \\
\text { saturation \% }\end{array}$} & 1 & 54 & $19.1 \pm 5.3$ & 17.7 & 20.6 & 8.6 & 30 & \multirow{5}{*}{ NS } \\
\hline & 2 & 3 & $21.8 \pm 7.8$ & 2.5 & 41 & 13.8 & 29.4 & \\
\hline & 3 & 18 & $19.7 \pm 8.4$ & 15.6 & 23.9 & 10 & 37.5 & \\
\hline & 4 & 49 & $19.5 \pm 9.4$ & 16.8 & 22.2 & 7.6 & 60 & \\
\hline & Total & 124 & $19.4 \pm 7.6$ & 18.1 & 20.8 & 7.6 & 60 & \\
\hline \multirow{5}{*}{$\mathrm{ALP}^{\ddagger}(\mathrm{U} / \mathrm{L})$} & 1 & 54 & $84.1 \pm 30.7$ & 75.7 & 92.5 & 36 & 176 & \multirow{5}{*}{ NS } \\
\hline & 2 & 3 & $85.7 \pm 44.2$ & 24.2 & 195.6 & 39 & 127 & \\
\hline & 3 & 18 & $95.8 \pm 39$ & 76.4 & 115.2 & 44 & 203 & \\
\hline & 4 & 49 & $80.7 \pm 30.9$ & 71.8 & 89.5 & 22 & 198 & \\
\hline & Total & 124 & $84.5 \pm 32.3$ & 78.7 & 90.2 & 22 & 203 & \\
\hline \multirow{5}{*}{$\mathrm{SGOT}^{\S}(\mathrm{U} / \mathrm{L})$} & 1 & 54 & $16.8 \pm 12.8$ & 13.3 & 20.3 & 5 & 73 & \multirow{5}{*}{$\begin{array}{l}F=2.5 \\
P<0.05\end{array}$} \\
\hline & 2 & 3 & $10.3 \pm 1.5$ & 6.5 & 14.1 & 9 & 12 & \\
\hline & 3 & 18 & $18.8 \pm 9.6$ & 14.0 & 23.6 & 5 & 40 & \\
\hline & 4 & 49 & $13.1 \pm 6.2$ & 11.3 & 14.8 & 6 & 32 & \\
\hline & Total & 124 & $15.5 \pm 10.2$ & 13.6 & 17.3 & 5 & 73 & \\
\hline \multirow{5}{*}{ SGPT"(U/L) } & 1 & 54 & $22.4 \pm 16.3$ & 18.0 & 26.9 & 7 & 79 & \multirow{5}{*}{$\begin{array}{l}F=2.5 \\
P<0.05\end{array}$} \\
\hline & 2 & 3 & $10 \pm 6.1$ & 5.1 & 25.1 & 6 & 17 & \\
\hline & 3 & 18 & $25.6 \pm 15.9$ & 17.6 & 33.5 & 8 & 64 & \\
\hline & 4 & 49 & $17.1 \pm 14.4$ & 12.9 & 21.2 & 5 & 87 & \\
\hline & Total & 124 & $20.5 \pm 15.6$ & 17.7 & 23.2 & 5 & 87 & \\
\hline
\end{tabular}

${ }^{\dagger}$ not significant; łalkaline phosphatase; §glutamic oxaloacetic transaminase; Tglutamic pyruvic transaminase

Table 3 Basic laboratory tests 2

\begin{tabular}{|c|c|c|c|c|c|c|c|c|}
\hline \multirow{2}{*}{ Item } & \multirow{2}{*}{ Group } & \multirow{2}{*}{$\mathbf{N}$} & \multirow{2}{*}{$\bar{X} \pm \mathbf{S D}$} & \multicolumn{2}{|c|}{ 95\% Interval } & \multirow{2}{*}{ Min } & \multirow{2}{*}{ Max } & \multirow{2}{*}{$\begin{array}{l}\text { F-test } \\
\text { P }\end{array}$} \\
\hline & & & & Upper & Lower & & & \\
\hline \multirow{6}{*}{$\begin{array}{l}\text { Serum urea } \\
(\mathrm{mmol} / \mathrm{l})\end{array}$} & 1 & 54 & $28,6 I \pm 6,03$ & 26,96 & 30,25 & 14,90 & 40,90 & \multirow{5}{*}{$\mathrm{NS}^{\dagger}$} \\
\hline & 2 & 3 & $24,30 \pm 7,34$ & 6,06 & 42,54 & 19,10 & 32,70 & \\
\hline & 3 & 18 & $26,72 \pm 4,61$ & 24,43 & 29,01 & 16,40 & 32,40 & \\
\hline & 4 & 49 & $29,72 \pm 5,15$ & 28,24 & 31,20 & 14,70 & 42,00 & \\
\hline & Total & 124 & $28,67 \pm 5,59$ & 27,67 & 29,66 & 14,70 & 42,00 & \\
\hline & I & 54 & $360.5 \pm 91.7$ & 335.5 & 385.5 & 127 & 583 & \multirow{5}{*}{ NS } \\
\hline \multirow{2}{*}{ Uric acid } & 2 & 3 & $416 \pm 52.9$ & 284.6 & 547.4 & 376 & 476 & \\
\hline & 3 & 18 & $332.2 \pm 77.8$ & 293.5 & 370.8 & 154 & 470 & \\
\hline \multirow[t]{4}{*}{ (umol/l) } & 4 & 49 & $347.1 \pm 94$ & 320.1 & 374.1 & 105 & 570 & \\
\hline & Total & 124 & $352.4 \pm 90.3$ & 336.4 & 368.5 & 105 & 583 & \\
\hline & I & 53 & $5.5 \pm 1.8$ & 5 & 6 & 3 & 10.8 & \multirow{5}{*}{ NS } \\
\hline & 2 & 3 & $6.9 \pm 3.5$ & 1.7 & 15.5 & 4.6 & 10.9 & \\
\hline \multirow{3}{*}{$\begin{array}{l}\text { Blood glucose } \\
(\mathrm{mmol} / \mathrm{L})\end{array}$} & 3 & 18 & $5.1 \pm 2.3$ & 3.9 & 6.2 & 2.3 & 11.5 & \\
\hline & 4 & 49 & $5.4 \pm 1.7$ & 4.9 & 5.9 & 1.4 & 13.4 & \\
\hline & Total & 123 & $5.4 \pm 1.9$ & 5.1 & 5.7 & 1.4 & 13.4 & \\
\hline
\end{tabular}




\begin{tabular}{|c|c|c|c|c|c|c|c|c|}
\hline \multirow{2}{*}{ Item } & \multirow{2}{*}{ Group } & \multirow{2}{*}{$\mathbf{N}$} & \multirow{2}{*}{$\bar{X} \pm \mathbf{S D}$} & \multicolumn{2}{|c|}{ 95\% Interval } & \multirow{2}{*}{ Min } & \multirow{2}{*}{ Max } & \multirow{2}{*}{$\begin{array}{l}\text { F-test } \\
P\end{array}$} \\
\hline & & & & Upper & Lower & & & \\
\hline \multirow{5}{*}{$\begin{array}{l}\text { Serum } \mathrm{Na}^{+} \\
(\mathrm{mmol} / \mathrm{l})\end{array}$} & I & 54 & $139.1 \pm 2.5$ & I 38.4 & 139.7 & 135 & 145 & \multirow{5}{*}{$\begin{array}{l}F=2.8 \\
P<0.043\end{array}$} \\
\hline & 2 & 3 & $\mid 38.7 \pm 1.5$ & 134.9 & 142.5 & 137 & 140 & \\
\hline & 3 & 18 & $138.3 \pm 3$ & 136.8 & 139.8 & 135 & 146 & \\
\hline & 4 & 49 & $137.3 \pm 3.5$ & 136.4 & 138.3 & 130 & 145 & \\
\hline & Total & 124 & $138.3 \pm 3.1$ & 137.7 & 138.8 & 130 & 146 & \\
\hline \multirow{5}{*}{$\begin{array}{l}\text { Serum } \mathrm{Cl}^{-} \\
(\mathrm{mmol} / \mathrm{l})\end{array}$} & I & 54 & $99.7 \pm 1.7$ & 99 & 99.9 & 95 & 103 & \multirow{5}{*}{$\begin{array}{l}F=2.9 \\
P<0.035\end{array}$} \\
\hline & 2 & 3 & $99.7 \pm 0.6$ & 98.2 & I0I.I & 99 & 100 & \\
\hline & 3 & 18 & $98.7 \pm 2.3$ & 97.6 & 99.9 & 95 & 105 & \\
\hline & 4 & 49 & $98 . \pm 2.6$ & 97.5 & 99 & 92 & 103 & \\
\hline & Total & 124 & $98.9 \pm 2.3$ & 98.5 & 99.3 & 92 & 105 & \\
\hline \multirow{5}{*}{$\begin{array}{l}\text { Serum } \mathrm{K}^{+} \\
(\mathrm{mmol} / \mathrm{l})\end{array}$} & 1 & 54 & $5.3 \pm 0.8$ & 5.1 & 5.5 & 3.7 & 6.7 & \multirow{5}{*}{ NS } \\
\hline & 2 & 3 & $5.2 \pm 0.4$ & 4.4 & 6.1 & 4.9 & 5.6 & \\
\hline & 3 & 18 & $5.4 \pm 0.7$ & 5 & 5.7 & 3.6 & 6.5 & \\
\hline & 4 & 49 & $5.5 \pm 0.9$ & 5.3 & 5.8 & 3.6 & 7.3 & \\
\hline & Total & 124 & $5.4 \pm 0.8$ & 5.3 & 5.5 & 3.6 & 7.3 & \\
\hline
\end{tabular}

tnot significant

Table 4 Malnutrition and inflammation parameters I

\begin{tabular}{|c|c|c|c|c|c|c|c|c|}
\hline \multirow{2}{*}{ Item } & \multirow{2}{*}{ Group } & \multirow{2}{*}{$\mathbf{N}$} & \multirow{2}{*}{$\bar{X} \pm \mathbf{S D}$} & \multicolumn{2}{|c|}{ 95\% Interval } & \multirow{2}{*}{ Min } & \multirow{2}{*}{$\operatorname{Max}$} & \multirow{2}{*}{$\begin{array}{l}\text { F-test } \\
\text { P }\end{array}$} \\
\hline & & & & Upper & Lower & & & \\
\hline \multirow{5}{*}{ Leukocyte count } & I & 54 & $6 \pm 1.9$ & 5.5 & 6.6 & 2.6 & 11.8 & \multirow{5}{*}{$\mathrm{NS}^{\dagger}$} \\
\hline & 2 & 3 & $7.6 \pm 0.8$ & 5.6 & 9.6 & 6.8 & 8.4 & \\
\hline & 3 & 18 & $6.0 \pm 1.8$ & 5.2 & 6.9 & 3.1 & 10.4 & \\
\hline & 4 & 49 & $5.7 \pm 1.8$ & 5.2 & 6.2 & 3.5 & 12.8 & \\
\hline & Total & 124 & $5.9 \pm 1.8$ & 5.6 & 6.3 & 2.6 & 12.8 & \\
\hline \multirow{5}{*}{$\begin{array}{l}\text { Serum creatinine } \\
\text { (umol/l) }\end{array}$} & I & 54 & $1021.9 \pm 214.2$ & 963.4 & 1080.4 & 578 & 1605 & \multirow{5}{*}{ NS } \\
\hline & 2 & 3 & $983 \pm 165.5$ & 571.9 & I394.I & 882 & 1174 & \\
\hline & 3 & 18 & $1020.4 \pm 246.1$ & 898 & II42.7 & 565 & 1483 & \\
\hline & 4 & 49 & $947.3 \pm 173.5$ & 897.5 & 997.2 & 614 & 1332 & \\
\hline & Total & 124 & $991.3 \pm 204.1$ & 955 & 1027.5 & 565 & 1605 & \\
\hline \multirow{5}{*}{ Total cholesterol } & I & 54 & $4.8 \pm 1.2$ & 4.5 & 5.1 & 2.6 & 7.5 & \multirow{5}{*}{ NS } \\
\hline & 2 & 3 & $4.6 \pm 1.3$ & 1.3 & 7.9 & 3.1 & 5.7 & \\
\hline & 3 & 18 & $4.4 \pm 1.2$ & 3.8 & 5 & 2.7 & 6.8 & \\
\hline & 4 & 49 & $4.6 \pm 1$ & 4.3 & 4.9 & 2.6 & 7.8 & \\
\hline & Total & 124 & $4.7 \pm I .1$ & 4.5 & 4.9 & 2.6 & 7.8 & \\
\hline \multirow{5}{*}{ LDL/HDL ${ }^{\ddagger}$} & I & 54 & $3.4 \pm 1.8$ & 2.9 & 3.9 & I & 11.5 & \multirow{5}{*}{ NS } \\
\hline & 2 & 3 & $2.2 \pm 2$ & 2.7 & 7.1 & 0.1 & 4.1 & \\
\hline & 3 & 18 & $2.7 \pm 1.9$ & 1.8 & 3.7 & 0.3 & 7.0 & \\
\hline & 4 & 49 & $3 \pm 1.5$ & 2.6 & 3.4 & 0.8 & 7.2 & \\
\hline & Total & 124 & $3.1 \pm 1.7$ & 2.8 & 3.4 & 0.1 & 11.5 & \\
\hline
\end{tabular}


Table Continues...

\begin{tabular}{|c|c|c|c|c|c|c|c|c|}
\hline \multirow{2}{*}{ Item } & \multirow{2}{*}{ Group } & \multirow{2}{*}{$\mathbf{N}$} & \multirow{2}{*}{$\bar{X} \pm \mathbf{S D}$} & \multicolumn{2}{|c|}{ 95\% Interval } & \multirow{2}{*}{ Min } & \multirow{2}{*}{$\operatorname{Max}$} & \multirow{2}{*}{$\begin{array}{l}\text { F-test } \\
\text { P }\end{array}$} \\
\hline & & & & Upper & Lower & & & \\
\hline \multirow{5}{*}{$\begin{array}{l}\mathrm{Ca} \times \mathrm{P}^{\S}\left(\mathrm{mmol}^{2} /\right. \\
\left.\mathrm{L}^{2}\right)\end{array}$} & I & 54 & $3.5 \pm 0.9$ & 3.3 & 3.8 & 1.7 & 5.8 & \multirow{5}{*}{ NS } \\
\hline & 2 & 3 & $2.9 \pm 1$ & 0.5 & 5.3 & 2.2 & 4.0 & \\
\hline & 3 & 18 & $3.2 \pm 1$ & 2.7 & 3.7 & 1.3 & 4.7 & \\
\hline & 4 & 49 & $3.3 \pm 0.8$ & 3.1 & 3.5 & 1.8 & 5.1 & \\
\hline & Total & 124 & $3.4 \pm 0.9$ & 3.2 & 3.5 & 1.3 & 5.8 & \\
\hline \multirow[b]{3}{*}{ iPTH" } & I & 53 & $60.8 \pm 96.9$ & 34.1 & 87.5 & 1.7 & 648 & \multirow{5}{*}{ NS } \\
\hline & 2 & 3 & $44.8 \pm 18.4$ & 20.8 & 90.5 & 28.3 & 64.6 & \\
\hline & 3 & 16 & $155.2 \pm 4 \mid 6.5$ & 66.7 & 377.1 & 5.3 & 1694.8 & \\
\hline \multirow[t]{2}{*}{ (pg/mL) } & 4 & 49 & $89.9 \pm 145.1$ & 48.2 & 131.6 & 0.7 & 578 & \\
\hline & Total & 121 & $84.7 \pm 187.5$ & 50.9 & 118.4 & 0.72 & 1694.8 & \\
\hline \multirow{5}{*}{$\begin{array}{l}\text { Charlson } \\
\text { comorbidity } \\
\text { index }\end{array}$} & I & 54 & $5.72 \pm 1.78$ & 5.2 & 6.2 & 3 & 12 & \multirow{5}{*}{ NS } \\
\hline & 2 & 3 & $5.33 \pm 1.15$ & 2.5 & 8.2 & 4 & 6 & \\
\hline & 3 & 18 & $5.39 \pm 1.19$ & 4.8 & 6 & 3 & 7 & \\
\hline & 4 & 49 & $5.55 \pm 1.36$ & 5.2 & 5.9 & 4 & 10 & \\
\hline & Total & 124 & $5.6 \pm 1.52$ & 5.3 & 5.9 & 3 & 12 & \\
\hline
\end{tabular}

${ }^{\dagger}$ not significant; ${ }^{\ddagger}$ atherogenic (LDL/HDL) index; ${ }^{\S}$ calcium-phosphate product; ${ }^{\top i n}$ tact parathyroid hormone.

Table 5Malnutrition and inflammation parameters 2

\begin{tabular}{|c|c|c|c|c|c|c|c|c|}
\hline \multirow{2}{*}{ Items } & \multirow{2}{*}{ Groups } & \multirow{2}{*}{$\mathbf{N}$} & \multirow{2}{*}{$\bar{X} \pm \mathbf{S D}$} & \multicolumn{2}{|c|}{ 95\% Interval } & \multirow{2}{*}{ Min } & \multirow{2}{*}{$\operatorname{Max}$} & \multirow{2}{*}{$\begin{array}{l}\text { F-test } \\
\text { P }\end{array}$} \\
\hline & & & & Upper & Lower & & & \\
\hline \multirow{5}{*}{$\begin{array}{l}\text { Body Mass Index } \\
\left(\mathrm{kg} / \mathrm{m}^{2}\right)\end{array}$} & I & 54 & $23.8 \pm 3.2$ & 23 & 24.7 & 17.2 & 30.9 & \multirow{5}{*}{$\begin{array}{l}F=10.1 \\
P<0.0001\end{array}$} \\
\hline & 2 & 3 & $27.1 \pm 4,6$ & 15.6 & 38.6 & 23.6 & 32.3 & \\
\hline & 3 & 18 & $21.1 \pm 1.8$ & 20.2 & 22 & 17.4 & 25.2 & \\
\hline & 4 & 49 & $21.4 \pm 2.8$ & 20.6 & 22.2 & 15.8 & 28.7 & \\
\hline & Total & 124 & $22.5 \pm 3.2$ & 22 & 23.1 & 15.8 & 32.3 & \\
\hline \multirow{5}{*}{$\begin{array}{l}\text { Serum albumin } \\
(\mathrm{g} / \mathrm{l})\end{array}$} & I & 53 & $40.8 \pm 3.4$ & 39.8 & 41.7 & 25 & 47 & \multirow{5}{*}{$\mathrm{NS}^{\dagger}$} \\
\hline & 2 & 3 & $40 \pm 2$ & 35 & 45 & 38 & 42 & \\
\hline & 3 & 18 & $4 I .1 \pm 2.2$ & 39.9 & 42.2 & 38 & 45 & \\
\hline & 4 & 49 & $40.2 \pm 2.2$ & 39.6 & 40.9 & 35 & 45 & \\
\hline & Total & 123 & $40.6 \pm 2.8$ & 40.1 & 41.1 & 25 & 47 & \\
\hline \multirow{5}{*}{$\begin{array}{l}\text { Serum ferritin } \\
(\mu \mathrm{mol} / \mathrm{l})\end{array}$} & 1 & 53 & $587.6 \pm 257.6$ & 516.6 & 658.6 & 91.5 & 1000 & \multirow{5}{*}{ NS } \\
\hline & 2 & 3 & $472 \pm 382.9$ & 479.3 & 1423.3 & 150.3 & 895.6 & \\
\hline & 3 & 18 & $477.9 \pm 236.7$ & 360.3 & 595.7 & 212.1 & 1000 & \\
\hline & 4 & 47 & $617.9 \pm 308.2$ & 527.4 & 708.3 & 19.8 & 1000 & \\
\hline & Total & $|2|$ & $580.2 \pm 279.2$ & 529.9 & 630.4 & 19.8 & 1000 & \\
\hline \multirow{5}{*}{$\begin{array}{l}\text { C reactive protein } \\
(\mu \mathrm{g} / \mathrm{dl})\end{array}$} & I & 49 & $4.9 \pm 6.9$ & 2.9 & 6.9 & 0.1 & 40.9 & \multirow{5}{*}{ NS } \\
\hline & 2 & 3 & $3.9 \pm 2.8$ & 3.2 & 10.9 & 1.8 & 7.1 & \\
\hline & 3 & 17 & $11.8 \pm \mid 9.1$ & 2 & 21.7 & 0.2 & 61.2 & \\
\hline & 4 & 48 & $5.7 \pm 9.2$ & 3 & 8.3 & 0.1 & 41.2 & \\
\hline & Total & 117 & $6.2 \pm 10.5$ & 4.3 & 8.1 & 0.1 & 61.2 & \\
\hline
\end{tabular}




\begin{tabular}{|c|c|c|c|c|c|c|c|c|}
\hline \multirow{2}{*}{ Items } & \multirow{2}{*}{ Groups } & \multirow{2}{*}{$\mathbf{N}$} & \multirow{2}{*}{$\bar{X} \pm \mathbf{S D}$} & \multicolumn{2}{|c|}{ 95\% Interval } & \multirow{2}{*}{ Min } & \multirow{2}{*}{$\operatorname{Max}$} & \multirow{2}{*}{$\begin{array}{l}\text { F-test } \\
\text { P }\end{array}$} \\
\hline & & & & Upper & Lower & & & \\
\hline \multirow{5}{*}{$\begin{array}{l}\text { Malnutrition- } \\
\text { inflammation } \\
\text { score }\end{array}$} & I & 54 & $5.8 \pm 3.2$ & 5 & 6.7 & 1 & 17 & \multirow{5}{*}{ NS } \\
\hline & 2 & 3 & $5 \pm 2$ & 0 & 10 & 3 & 7 & \\
\hline & 3 & 18 & $6 \pm 3.2$ & 4.4 & 7.6 & 2 & 15 & \\
\hline & 4 & 49 & $6.4 \pm 2.6$ & 5.6 & 7.1 & 2 & 14 & \\
\hline & Total & 124 & $6 \pm 2.9$ & 5.5 & 6.6 & I & 17 & \\
\hline
\end{tabular}

tnot significant

Table Abbreviations: NS:Not Significant;AP:Alkaline Phosphatase; SGOT: Glutamic Oxaloacetic Transaminase;SGPT:Glutamic Pyruvic Transaminase;LDL/ HDL:Atherogenicindex; Ca $\times$ P:Alcium-Phosphate Product; $\mathrm{PTH}$ :Intact Parathyroid Hormone

SGOT and SGPT values were within reference range; however, a statistically significant difference was noted between the groups ( $p$ $<0.05$, Table 2). ALP (Table 2) and serum albumin (Table 5) levels were within reference range, with no significant differences between groups. Serum potassium levels did not differ significantly between groups, but serum sodium and chloride did $(\mathrm{p}<0.043$ and $\mathrm{p}<0.035$ respectively). Patients with lowest volume overload (group 1) had highest sodium and chloride levels, while patients with highest volume overload (group 4) had lowest sodium and chloride levels (Table 3).

Uric acid, blood glucose (above the upper threshold), leukocyte count,serum creatinine, total cholesterol, atherogenicity index (LDL/ HDL), iPTH, calcium-phosphate product and CCI scores did not differ significantly between groups (Table 3,4). CRP levels did not differ significantly between groups, even though highest means were recorded in group 3 (Table 5). It must be noted, however, that these means were accompanied by rather high standard deviations (Table 5). Patients in group 3 had lowest BMI ( $<<0.0001$, Table 5).

Patients in group 4, with high volume overload, had highest MIS, while those in group 2, with lower volume overload, had lowest MIS, but the difference was not statistically significant (Table 5).Still, it should be noted that patient's distribution was such that this group was much smaller then the other three (only 3 patient's vs 54, 18 and 49 in groups 1, 3 and 4 respectively).According to the chosen parameters, the highest prevalence of MICS was found in group 4 (Figure 2). Patients in group 4 had three times higher chances to have MICS then those in group 3 Odds Ratio $(\mathrm{OR})=3$, confidence interval (CI): $1.7645<$ O.R. $<5.1007$. Relative risk of MICS in group 4 was 2.8 times greater then in group 3 .

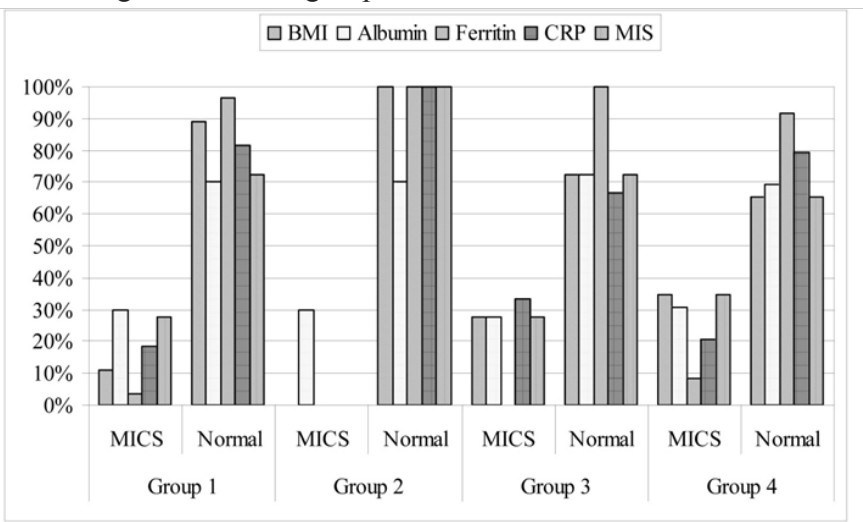

Figure 2Chosen parameters of malnutrition inflammation complex syndrome (MICS).

MICS: Body Mass Index (BMI) $<20 \mathrm{~kg} / \mathrm{m}^{2}$, Albumin $<40 \mathrm{~g} / \mathrm{L}$, Ferritin $\geq 1000$ umol/l, CRP $>8 \mathrm{ug} / \mathrm{dl}$ and Malnutrition-inflammation Score (MIS) $>8$.

Normal: BMI $\geq 20 \mathrm{~kg} / \mathrm{m}^{2}$, Albumin $\geq 40 \mathrm{~g} / \mathrm{L}$, Ferritin $<1000$ umol/l, CRP $\leq 8$ ug/ $\mathrm{dl}$ and $\mathrm{MIS} \leq 8$.
ROC analysis demonstrated that in combination of all five investigated parameters the strongest predictor of MICS in groups 1 and 4 was BMI (Area $=0.699, \mathrm{SE}=0.049, \mathrm{p}=0.000,95 \% \mathrm{CI}=0.603$ - 0.795 andArea $=0.329, \mathrm{SE}=0.052, \mathrm{p}=0.002,95 \% \mathrm{CI}=0.227$. 0.432 respectively), followed by MIS (group 1: Area $=0.399, \mathrm{SE}=$ $0.054, \mathrm{p}=0.053,95 \% \mathrm{CI}=0.301-0.512$; group 4: Area $=0.612, \mathrm{SE}$ $=0.054, \mathrm{p}=0.057,95 \% \mathrm{CI}=0.496-0.707)$ (Figure 3). The strongest predictor of MICS in group 3 was serum ferritin (Area $=0.327, \mathrm{SE}=$ $0.063, \mathrm{p}=0.023,95 \% \mathrm{CI}=0.205-0.45$ ), followed by BMI (Area $=$ $0.357, \mathrm{SE}=0.059, \mathrm{p}=0.051,95 \% \mathrm{CI}=0.252-0.483$ ). Serum albumin and CRP followed, but with no statistically significant sensitivity and specificity. Small number of patients in group 2 precluded testing for sensitivity and specificity of the chosen parameters of MICS in this group.

\section{ROC Curve}

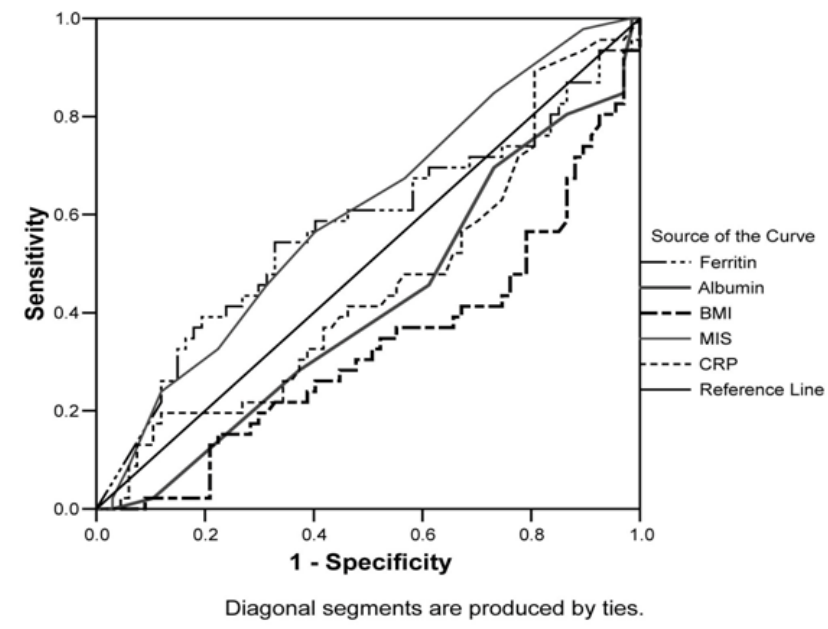

Figure 3 Sensitivity and specificity of the chosen parameters of malnutrition inflammation complex syndrome (MICS) in patients with interdialytic weight gain/total body water $>5 \%$ and interdialytic weight gain/dry body weight $>10 \%$.

\section{Discussion}

MICS is undoubtedly present in ESRD patients even before the beginning of $\mathrm{HD},{ }^{1-4}$ but dialysis introduces new entities which might contribute to it: contact of blood with bio-incompatible materials, presence of impurities in dialysate, artificial vascular access, limited clearance of bioactive pro-inflammatory substances, altered dietary regime to avoid catabolism, loss of muscular mass and the risk of hyperhydration. ${ }^{2-4}$ The discontinuous nature of HD causes sawtooth volume fluctuations. ${ }^{9}$ During HD, the plasma compartment is ultrafiltered down to a nadir and refills from the interstitial space several hours after dialysis, causing remarkable volume oscillations

Citation: Vlatković , Trbojević-Stanković J, Stojimirović B. Relationship between malnutrition-inflammation complex syndrome and fluid balance in maintenance hemodialysis patients. Urol Nephrol Open Access J. 20 I7;4(5): I 58-I67. DOI: I0.15406/unoaj.2017.04.00 I44 
in the vascular system of dialysis patients. High IDWG, caused by significant salt and water intake between dialysis sessions, elicits left ventricular dilatation and increased sympathetic activity, resulting in myocardial cells production of inflammatory markers. ${ }^{18}$ Continuous maintaining of adequate DBW in HD patients significantly contributes to control of hypervolemia and prevents myocardial alterations related to chronic inflammation.?

Individual features of $\mathrm{HD}$ patients such as age, residual diuresis, BMI and TBW have substantial influence on fluid balance maintenance. The same IDWG may result in different volume load depending on these characteristics. For this reason we believe that IDWG as a single parameter of volume overload is not accurate enough. Therefore, in this study we opted to distribute patients according to IDWG vs. DBW and TBW ratios (Figure 1). Such distribution better depicts patients' compliance with diet and much better reflects relative volume overload. Water and sodium retention are the most important factors for volume disturbance and arterial hypertension in HD patients. ${ }^{5,6}$ Sodium distribution in body fluid compartments is not homogeneous. About $90 \%$ of exchangeable sodium is in the extracellular compartment and only $10 \%$ in the intracellular. ${ }^{9}$ Serum sodium concentration is inversely related to TBW; therefore high sodium levels designate low TBW, while lower sodium is found in patients with higher TBW. ${ }^{12}$ In the current study, highest sodium and chloride levels were found in patients with lowest volume overload, while the lowest levels were recorded in patients with highest volume overload, due to dilution. Even though these findings correlate well with literature data, such sodium and volume relations did not significantly influence BP in our patients, as reported by other authors. ${ }^{6,10,11}$ We believe that most patients in our study had satisfactory volume regulation, but the effect of antihypertensive therapy should not be undermined. Despite different fluid status of the groups, no statistically significant difference was found in HGB and HCT levels, and $\mathrm{Ca} \times \mathrm{P}$ product, indicating that supplementary therapies were effective (erythropoietin, phosphate binders).

No uniform approach to MICS evaluation has been defined so far. A number of different parameters and models may be used in assessing MICS presence..$^{2,4,5}$ Relatively wide range of MICS markers and their variable predictive value result in diversity in interpreting this syndrome. According to the chosen parameters of MICS in our study, the overall MICS prevalence was up to $30 \%$, and the highest incidence of MICS markers was found in patients with highest volume overload. Odds ratio analysis showed higher MICS prevalence in patients with lower BMI. The highest malnutrition-inflammation scores were recorded in patients with volume overload (Group 4). Sensitivity and specificity analysis identified BMI and MIS as the most sensitive markers of MICS in groups 1 and 4. Kalantar-Zadehet al. ${ }^{17}$ also identified MIS as useful, short-term tool to circumvent the need for measuring inflammation markers, CRP or IL6, and risk-stratify HD patients. However, in group 3, the most sensitive marker was serum ferritin, similar to findings by other authors. ${ }^{19}$ Patients in this group were youngest, had highest residual diuresis and high IDWG. They could tolerate higher IDWG, suggesting better nutritional status, with less volume overload, thanks to better preserved residual diuresis. All these features contributed to lower possibility for MICS presence in this group, as reported by other authors. ${ }^{11,20}$

IDWG is directly associated with calorie and protein intake and better nutrition status. ${ }^{1-4,21}$ It is speculated that the positive influence of IDWG on nutritional status is more prominent than the negative effects of hypervolemia and hypertension caused by high IDWG. ${ }^{11}$ In our study, the lowest incidence of MICS markers was recorded in patients in group 3. This result confirms positive effect of higher IDWG, since these patients are considered to be well nourished and have adequately controlled fluid balance. Nevertheless, a clear distinction should be made between high IDWG reflecting isolated dietary non-compliance as opposed to good nutritional status. Dietary recommendations for HD patients should be well tailored not to have negative influence on the quality of nutrition. Sodium intake certainly is the most important factor, but sodium dialysate concentration, saline infusions during HD or hyperglycemia in diabetic patients can also cause thirst. ${ }^{22,23}$ Volume overload is associated with adverse outcome in HD patients, so it is advisable to employ the recommended strategies for maintaining adequate fluid balance in these patients. ${ }^{9}$

\section{Conclusion}

Nearly one third of patients had increased majority of the MICS markers. High IDWG is associated with higher volume overload, which represents an additional risk for MICS. Patients with low BMI who do not comply with recommended diet and fluid intake are at highest risk of developing volume overload, which will then require higher ultrafiltration, thus additionally disturbing body fluid composition, and creating additional risk for MICS. BMI and MIS appeared to be the most sensitive predictors of MICS in our study population, while low BMI was the highest risk factor for volume disturbance.

\section{Acknowledgments}

None.

\section{Conflicts of interest}

None.

\section{References}

1. Pecoits-Filho R, Lindholm B, Stenvinkel P. The malnutrition, inflammation, and atherosclerosis (MIA) syndrome - the heart of the matter. Nephrol Dial Transplant. 2002;17(supp 11):28-31.

2. Kalantar-Zadeh K, Ikizler TA, Block G, et al. Malnutrition-inflammation complex syndrome in dialysis patients: causes and consequences. Am J Kidney Dis. 2003;42(5):864-881.

3. Beddhu S, Pappas LM, Ramkumar N, et al. Malnutrition and atherosclerosis in dialysis patients. J A Soc Nephrol. 2004;15(3):733-742.

4. Nascimento MM, Pecoits-Filho R, Lindholm B, et al. Inflammation, malnutrition and atherosclerosis in end-stage renal disease: a global perspective. Blood Purif. 2002;20(5):454-458.

5. Goncalves S, Pecoits-Filho R, Perreto S, et al. Association between renal function, volume status and endotoxaemia in chronic kidney disease patients. Nephrol Dial Transplant. 2006;21(10):2788-2794.

6. Kooman JP, van der Sande FM, Leunissen KML. Sodium, blood pressure and cardiovascular pathology: is it all volaemia? Nephrol Dial Transplant. 2004;19(5):1046-1049.

7. Kuhlmann MK, Levin NW. Interaction between nutrition and inflammation in hemodialysis patients. Contrib Nephrol. 2005;149:200-207.

8. Bayes B, Pastor MC, Bonal J, et al. Oxidative stress, inflammation and cardiovascular mortality in haemodialysis - role of seniority and intravenous ferrotherapy: analysis at 4 years of follow-up. Nephrol Dial Transplant. 2006;21(4):984-990. 
9. Charra B. Fluid balance, dry weight, and blood pressure in dialysis. Hemodial Int. 2007;11(1):21-31.

10. Movilli E, Cancarini GC, Cassamali S, et al. Inter-dialytic variations in blood volume and total body water in uraemic patients treated by dialysis. Nephrol Dial Transplant. 2004;19(1):185-189.

11. Lopez-Gomez JM, Villaverde M, Jofre R, et al. Interdialytic weight gain as a marker of blood pressure, nutrition, and survival in hemodialysis patients. Kidney Int. 2005;67(93):S63-S68.

12. De Palma JR, Pittard JD. Body water-body weight (Part I). Dial Transplant. 2001;30(10):700-705.

13. De Palma JR, Pittard JD. Body mass index and uremia (Part I). Dial Transplant. 2002;31(5):342-350.

14. Horl WH, Vanrenterghem Y, Aljama P, et al. Optimal treatment of anemia in patients with chronic kidney disease (CKD). Nephrol Dial Transplant. 2007;22(suppl 31):iii20-iii26.

15. Fouque D, Vennegoor M, ter Wee P, et al. EBPG guideline on nutrition. Nephrol Dial Transplant. 2007;22(suppl 2):ii45-ii87.

16. Tattersall J, Martin-Malo A, Pedrini L, et al. EBPG guideline on dialysis strategies. Nephrol Dial Transplant. 2007;22(suppl 2):iii5-iii21.

17. Kalantar-Zadeh K, Kopple JD, Humpherys MH, et al. Comparing outcome predictability of markers of malnutrition-inflammation complex syndrome in haemodialysis patients. Nephrol Dial Transplant. 2004;19(6):1507-1519.

18. Stenvinkel P, Heimburger O, Kindholm B, et al. Are there two types of malnutrition in chronic renal failure? Evidence for relationships between malnutrition, inflammation and atherosclerosis (MIA syndrome). Nephrol Dial Transplant. 2000;15(7):953-960.

19. Bross R, Zitterkoph J, Pithia J, et al. Association of serum total ironbinding capacity and its changes over time with nutritional and clinical outcomes in hemodialysis patients. Am J Nepohrol. 2009;29(6):571-581.

20. Bargman JM, Golper TA. The importance of residual renal function for patients on dialysis. Nephrol Dial Transplant. 2005;20(4):671-673.

21. Beddhu S, Ramkumar N, Pappas LM. Normalization of protein intake by body weight and the associations of protein intake with nutritional status and survival. J Ren Nutr. 2005;15(4):387-397.

22. Oliver A, Wright M, Matson A, et al. Low sodium haemodialysis reduces interdialytic fluid consumption but paradoxically increases post-dialysis thirst. Nephrol Dial Transplant. 2004;19(11):2883-2885.

23. Sung J-M, Kuo S-C, Guo H-R, et al. The role of oral dryness in interdialytic weight gain by diabetic and non-diabetic haemodialysis patients. Nephrol Dial Transplant. 2006;21(9):2521-2528. 